\section{Sevelamer-associated ischemic colitis with perforation}

\author{
Rachel Hudacko, ${ }^{1,2}$ Peter Kaye ${ }^{2,3}$ \\ 1Orange Pathology Associates PC, \\ Middletown, NY; ${ }^{2}$ Good Samaritan \\ Regional Medical Center, Suffern, NY; \\ ${ }^{3}$ Ramapo Valley Surgical Associates, \\ Suffern, NY, USA
}

\section{Abstract}

We present a case of an 83-year-old woman with end-stage renal disease and hyperphosphatemia treated with sevelamer carbonate, who underwent subtotal colectomy for diffuse bowel necrosis and two perforations in the transverse colon. Histologic examination revealed ischemic colitis with crystals consistent with sevelamer carbonate embedded in ulcer debris and within the colonic wall in areas of transmural necrosis. This is a novel cause of drug-induced ischemic colitis with subsequent perforation that has not yet been reported in the literature. Clinicians and pathologists should be aware of the potential complications of sevelamer use and the histologic features of sevelamer-induced colonic injury.

\section{Introduction}

Sevelamer carbonate is an ion-exchange resin that binds phophate and is used to treat hyperphosphatemia due to chronic kidney disease. ${ }^{1}$ Common adverse effects include nausea, vomiting, diarrhea, constipation, and abdominal pain. A recent case series described the histologic features of sevelamer crystals in the gastrointestinal tract and suggested an association with mucosal injury. ${ }^{2}$ Subsequent cases of sevelamer-associated colonic mucosal injury presenting with hematochezia and of sevelamer-induced colitis with pseudotumor formation have been reported. ${ }^{3,4}$ We would like to add to this small body of literature by reporting a case of sevelamer-associated ischemic colitis with perforation.

\section{Case Report}

An 83 year old woman with a past medical history of diabetes mellitus, hypertension, atrial fibrillation, and hemodialysis-dependent end-stage renal disease presented to the emergency department unresponsive and febrile from an outside assisted-living facility. She was being treated with sevelamer carbonate, $1600 \mathrm{mg}$ three times daily, for hyperphosphatemia. On hospital day two, she became hypotensive requiring pressors and developed increasing abdominal distension. A surgical consult was obtained, and an abdominal x-ray showed subdiaphragmatic air. An exploratory laparotomy was performed for suspected perforated viscus. During the laparotomy, the colon appeared friable with patchy necrosis throughout, but more severe in the transverse colon. A subtotal colectomy with end-ileostomy was performed for suspected Clostridium difficileassociated pseudomembranous colitis. Subsequent blood cultures and Clostridium difficile DNA tests were negative. The patient had a prolonged post-operative course complicated by aspiration pneumonia and occlusion of the upper extremity dialysis vascular access with cellulitis. The patient was discharged on post-operative day fifteen.

On gross examination, the colonic mucosa was erythematous from the cecum down to 15 $\mathrm{cm}$ from the distal margin with multiple irregular ulcers (Figure 1). Two gross perforations were identified in the transverse colon. Pseudomembranes were not present. The serosa was dusky with patchy fibrinous exudate. Histologic examination revealed mucosal ischemic injury with ulcers, transmural necrosis, and acute serositis. Crystalline material morphologically consistent with sevelamer crystals (Figure 2) was noted in the ulcer exudate and intramurally in foci of transmural necrosis. Intact diverticula without associated diverticulitis were also present. The mesenteric/subserosal arteries showed intimal hyperplasia. There was no evidence of calcific atherosclerosis, thromboemboli, cholesterol emboli, or vasculitis.

\section{Discussion}

Sevelamer is an orally administered nonabsorbed phosphate-binding resin that has recently been reported to be associated with gastrointestinal tract injury including mucosal ischemia, ulcers, necrosis, and pseudotumor formation. ${ }^{1-4}$ The histologic features of sevelamer crystals were first described by Swanson et al. in a case series of upper and lower gastrointestinal tract biopsy and resection specimens and were validated by submitting crushed sevelamer tablets for histologic examination. ${ }^{2}$ They described the crystals as nonpolarizable with a broad irregularly-spaced fish scale pattern and a two-toned appearance consisting of bright pink accentuations with a rusty yellow background. These crystals were compared to two other common drug crystals identified in gastrointestinal specimens: sodium polystyrene sulfonate (translucent purple
Correspondence: Rachel Hudacko, Department of Pathology, Good Samaritan Regional Medical Center, 255 Lafayette Avenue, Suffern, NY 10901, USA.

Tel.: + 1.845.368.5529 - Fax: +1.845.368.5329.

E-mail: rhudacko@gmail.com

Key words: Sevelamer carbonate; Renvela; ischemic colitis; chronic kidney disease; phosphate.

Contributions: RH, concept and interpretation of data, drafting of article; PK, patient care, critical revision for intellectual content; $\mathrm{RH}, \mathrm{PK}$, final approval.

Conflict of interest: the authors declare no conflict of interest.

Received for publication: 21 July 2015.

Accepted for publication: 1 September 2015.

This work is licensed under a Creative Commons Attribution NonCommercial 3.0 License (CC BYNC 3.0).

(C) Copyright R. Hudacko and P. Kaye, 2015 Licensee PAGEPress, Italy

Gastroenterology Insights 2015; 6:6116

doi:10.4081/gi.2015.6116

crystals with regularly-spaced fish-scale pattern) and cholestyramine (opaque bright orange/magenta crystals without fish-scale pattern). They reported that the severity of the mucosal injury appeared to correlate with the sevelamer dosage and that gastrointestinal dysmotility may contribute to crystal concentration and local mucosal injury but concluded that larger studies should be performed to determine whether sevelamer is the actual cause of the injury or just a coincidental histologic finding. ${ }^{2}$

Subsequently, Chintamaneni et al. described a patient presenting with hematochezia who was found to have a $5 \mathrm{~cm}$ ulcer in the sigmoid colon. ${ }^{3}$ Biopsy revealed sevelamer crystals entrapped in the ulcer bed. Sevelamer was discontinued without further bleeding, and the authors postulated that sevelamer crystals are directly toxic to the colonic mucosa and may result in ischemic injury. ${ }^{3}$ Okwara et al. described a patient who presented with non-bloody diarrhea who was found to have a circumferential polypoid mass in the cecum. ${ }^{4}$ Biopsy showed sevelamer crystals in association with necrosis and ischemic changes without evidence of dysplasia or malignancy, and the authors concluded that the mass formation was the result of sevelamer-induced colitis. ${ }^{4}$

Herein, we describe the first case of a colon perforation due to sevelamer-associated ischemic colitis. Sevelamer crystals were found in the ulcer exudate and within the 
colonic wall in areas of transmural necrosis. We found no other pathologic cause for ischemic colitis.

Although the patient was hypotensive before surgery, the majority of the colon was involved with the most severely affected region being the transverse colon, as opposed to the water- shed regions (i.e. splenic flexure, rectosigmoid junction) as would be expected in ischemic colitis due to an acute decrease in blood flow. Although vascular pathology was not identified in the specimen, occlusion of larger more proximal mesenteric arteries (i.e. superior mesenteric, inferior mesenteric) cannot be

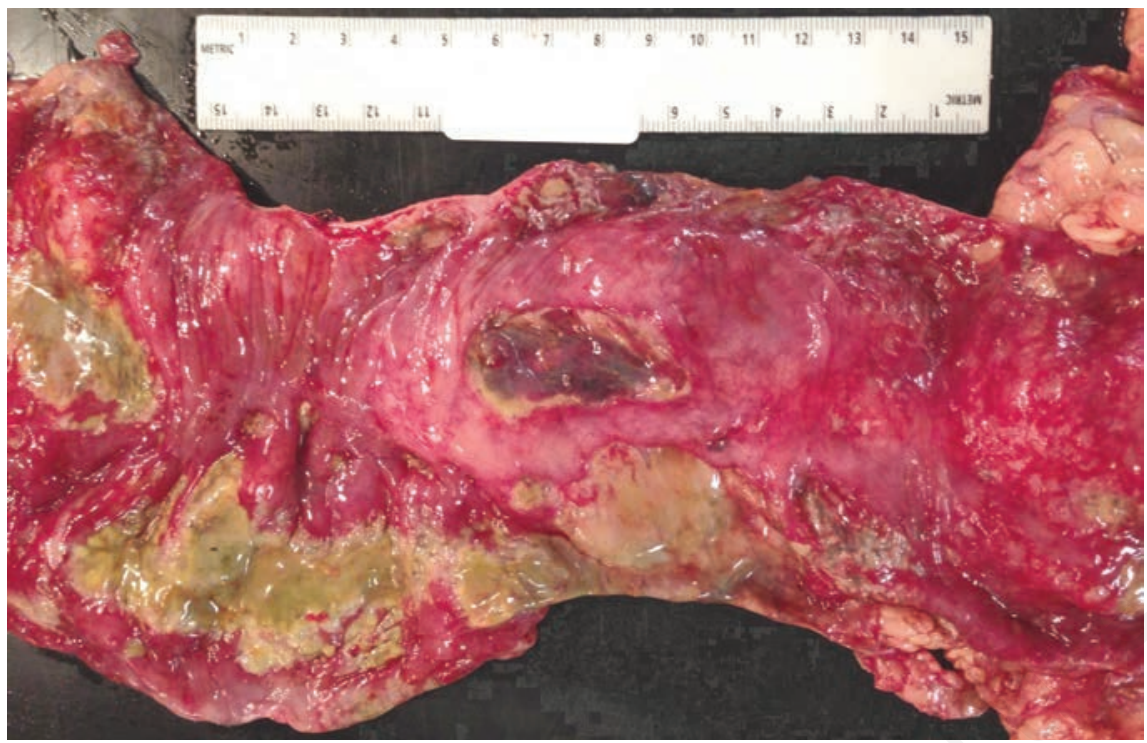

Figure 1. This segment of distal transverse colon shows an erythematous, granular mucosa with multiple irregular ulcers covered by tan-brown and focally hemorrhagic (center) exudate.

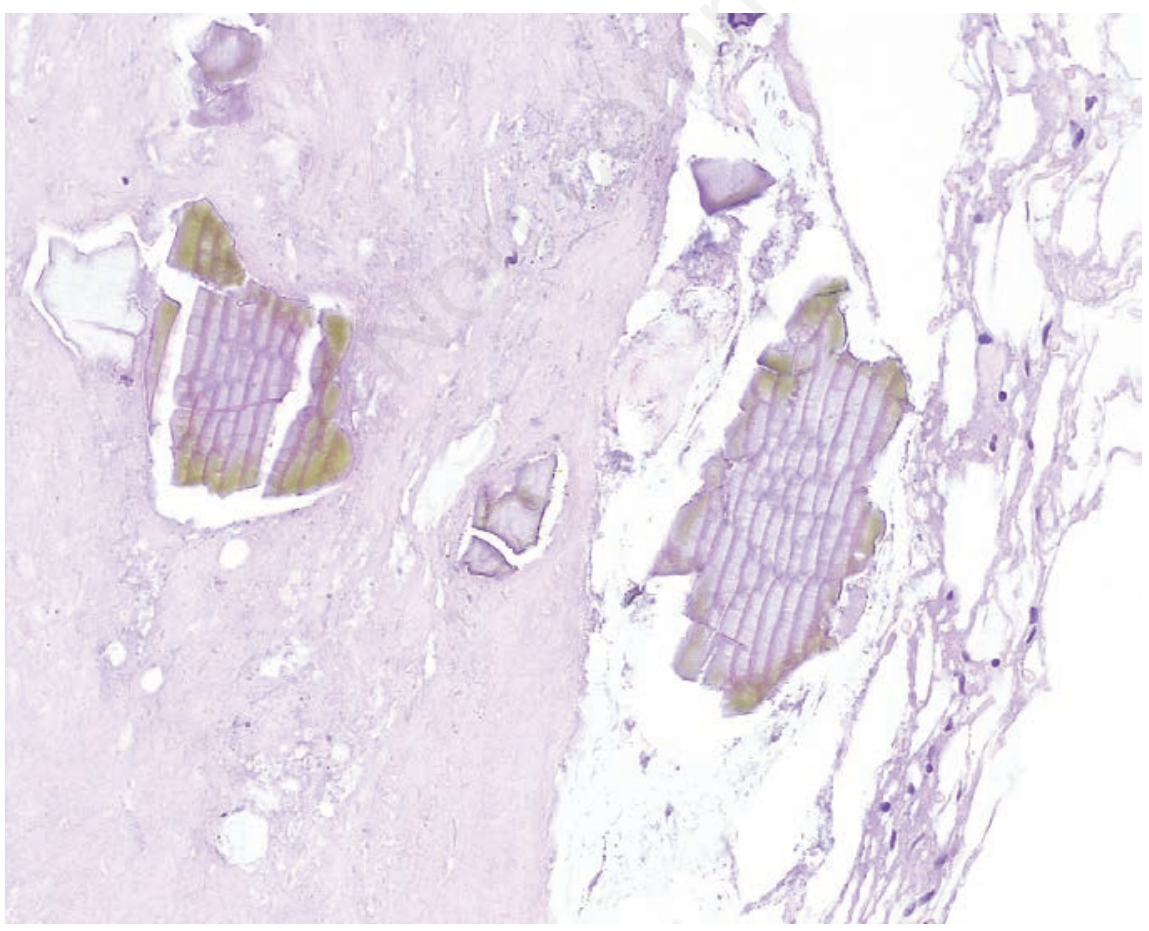

Figure 2. Sevelamer crystals present in the ulcer exudate are translucent and have a twotoned appearance with pink linear accentuations creating a fish-scale pattern and a yellow-gold periphery. completely excluded since neither a computed tomography scan nor angiography was performed. However, multiple vessels would have to be involved to affect the majority of the colon, which is less likely. Besides sevelamer, the patient had no history of recent use of other drugs typically associated with ischemic colitis (i.e. non-steroidals, sodium polystyrene sulfonate, cocaine, etc).

\section{Conclusions}

In summary, we present the first reported case of sevelamer-associated ischemic colitis with multiple perforations in a patient with end-stage renal disease. Although a causal role has yet to be established, it is important for clinicians, especially gastroenterologists and colorectal surgeons, to be aware of the possible gastrointestinal complications in order to prevent more severe consequences in patients with suspected colitis who are taking sevelamer. Pathologists also need to be aware of the morphologic features of sevelamer crystals and its mimics to aid in the diagnosis of such complications.

\section{References}

1. Perry CM, Ploster GL. Sevelamer carbonate: a review in hyperphosphataemia in adults with chronic kidney disease. Drugs 2014;74:771-92.

2. Swanson BJ, Limetkai BN, Liu TC, et al. Sevelamer crystals in the gastrointestinal tract (GIT): a new entity associated with mucosal injury. Am J Surg Pathol 2013;37:1686-93.

3. Chintamaneni P, Das R, Kuan SF, et al. Hematochezia associated with sevelamerinduced mucosal injury. ACG Case Rep J 2014;1:145-7.

4. Okwara C, Choi C, Park J. Sevelamerinduced colitis presenting as a pseudotumor. Clin Gastroenterol Hepatol 2015 [Epub ahead of print]. 